\title{
Du désordre au sentiment de sécurité
}

\section{From disorder to sense of security}

\author{
F. Molénat $\cdot$ C. Chanal \\ (C) Springer-Verlag France 2014
}

Fin des années 1990 un gynécologue-obstétricien parlait ainsi des situations de toxicomanie : "avant, c'était le désordre, et un sentiment d'impuissance généralisé ; maintenant tout se déroule dans le calme et la sécurité... " [1]. Il résumait en une phrase l'étonnant changement face à une situation jusqu'alors redoutée du monde périnatal, social, psychiatrique. Un suivi médical coordonné, centré sur l'objectif commun aux parents et aux professionnels de la naissance - donner les meilleures chances à l'enfant - opérait un tournant radical, avec peu de moyens supplémentaires mais une modification profonde des habitudes. Les résultats obstétricaux, pédiatriques, sociaux, psychologiques, ne se firent pas attendre [2]. On était passé d'une " toxicomane enceinte à une femme enceinte qui a un problème de toxicomanie » [1].

L'amélioration des indicateurs n'est pas passée par des techniques matérialisées, mais par une gestion rigoureuse des relations entre parents et professionnels et des divers intervenants entre eux. Des outils qualitatifs se dégagèrent, et l'échange d'expérience avec d'autres équipes a permis d'en vérifier la pertinence. Aux peurs mutuelles a succédé un climat de sécurité dont les effets thérapeutiques ne sont plus à démontrer. La mobilisation des représentations professionnelles s'est traduite par une transformation tant dans l'accueil des futures mères et des couples, que dans l'acceptation par ceux-ci du suivi proposé. Les femmes enceintes sont venues consulter de plus en plus tôt, réduisant la part d'imprévu source de confusion, et permettant d'utiliser les nouveaux outils « relationnels » en prise directe sur le suivi obstétricopédiatrique. Les aspects sociaux et psychologiques, amarrés aux consultations médicales, pouvaient se

\footnotetext{
F. Molénat $(\bowtie)$

Association de formation et de recherche sur l'enfant et son environnement, BP 64164,

F-34092 Montpellier cedex 5, France

e-mail : afree@afree.asso.fr

C. Chanal

CHU Arnaud-de-Villeneuve,

371, avenue du Doyen-Gaston-Giraud,

F-34295 Montpellier cedex 05, France
}

traiter avec une rigueur nouvelle, dans un partenariat étroit entre futurs parents et professionnels.

Si les principes simples, fondés sur une pierre d'angle accueil bienveillant ouvrant sur un suivi obstétrical resserré d'une femme enceinte souffrant d'addiction - ont produit un tel changement, il nous reste en mémoire cependant l'intense activité de certains qui fut nécessaire pour qu'évoluent les représentations des professionnels. $\mathrm{Si}$, du côté des futurs parents, un accueil médical bienveillant, sans jugement ni injonction, a suffi pour amorcer un retournement, cette « bienveillance » a nécessité le feutrage précis d'éléments de sécurité chez les professionnels de la naissance. Ces femmes au comportement difficile, inquiétantes, redevenaient des mères comme les autres, soucieuses du bien-être de leur enfant, qui se présentaient de plus en plus tôt dans la grossesse, accompagnées par le futur père lui-même sollicité par la sage-femme "référente ». La problématique « grossesse et addiction » devenait lisible, et rendait possible une prise en charge adaptée à chaque cas. Sortir de l'impuissance favorisait la qualité d'accueil, sans se leurrer sur la difficulté permanente à intégrer dans la culture médicale " classique » des éléments de fragilité qui sortent du champ strictement somatique et obligent à modifier les habitudes de travail.

La consommation d'alcool a résisté plus longtemps à une approche médicale sereine, pour les raisons exposées plus loin par l'équipe de Lille. Cependant, on peut noter un fait significatif : lorsque des pédiatres informèrent qu'un enfant à risque d'imprégnation pendant la vie fœtale aurait un meilleur devenir grâce à un soutien développemental précoce, il devint plus aisé à l'équipe obstétricale d'aborder le sujet grâce à un outil rassurant à proposer. Au-delà d'une aide possible en vue de diminuer les prises avant la naissance, la connaissance du problème permettrait de rencontrer le pédiatre en anténatal et d'anticiper un suivi adéquat. Ainsi, il ne s'agit pas tant de dépister que d'organiser les conditions de la confiance, pour que les facteurs de vulnérabilité, teintés négativement quant à l'image de soi, s'expriment et trouvent des réponses sécurisantes.

Simplicité n'est pas facilité. Avancer dans la complexité des risques médicaux tant chez la femme enceinte que chez 
le nouveau-né selon le type d'addiction, associés aux fragilités existentielles de la famille, le tout dans un climat de culpabilité intense chez les futures mères, donne toute leur valeur aux protocoles qui se sont peu à peu élaborés, avec l'aide des Réseaux périnatals [3]. Ceci exige une attention rigoureuse pour que les futurs parents, et chaque professionnel concerné, se maintiennent dans une cohérence et une lisibilité par tous du projet qui se construit progressivement, au fur et à mesure des rencontres entre famille et intervenants. La coordination, le « suivi du suivi », prennent leur place dans la prise en charge médicale, à des rythmes différents selon la dynamique institutionnelle de chaque terrain. Il est devenu banal d'utiliser la métaphore « rigueur chirurgicale » pour qualifier ce minutieux tissage de rencontres et de liaisons dont la solidité soutiendra un possible changement chez la femme enceinte : confiance en soi, confiance dans un professionnel, confiance dans le système de soins, restauration d'une image de soi souvent écornée par les aléas d'un parcours qui a conduit à l'addiction, seront les ingrédients d'une «nouvelle expérience de relation » chez les plus vulnérables. La clé de l'efficacité reste l'axe organisateur du suivi médical, fil lisible et continu tant pour les parents que pour les professionnels, non stigmatisant, à partir duquel se tissent les orientations nécessaires selon les cas. Enfin, la place essentielle du médecin généraliste dans la confiance et la continuité des soins s'en trouvait éclairée, fait nouveau dans les pratiques « classiques ».

Les « covulnérabilités » issues d'un contexte de vie précaire, parfois des troubles psychiques plus aigus, soulèvent des questions annexes : quel accueil social pour accompagner dans la durée des liens parents-enfant fragiles, quelles collaborations, quel suivi d'aval, quelle formation pour un esprit de travail qui sollicite de multiples acteurs peu habitués à coordonner leur action?

L'expérience montre que cette problématique, tant redoutée jusqu'aux années 1990, a donné l'occasion de construire une véritable sémiologie d'une clinique dite " en réseau ", c'est-à-dire le moyen de réintégrer dans le processus de naissance l'ensemble des composantes qui influeront sur le développement de l'enfant : conditions d'accueil de la parole des parents, prise en compte pas à pas de leur évolution émotionnelle, sécurité somatique par un suivi ajusté, réduction des facteurs de stress, continuité et cohérence des actions, anticipation du retour à domicile. Il devient possible d'intégrer dans un référentiel les nouveaux outils qui scellent sans contraindre un environnement humain souple, le propre sentiment de sécurité de chaque professionnel restant la condition incontournable de l'efficacité. Tout un vocabulaire, désormais banalisé, est né d'une volonté de sortir de l'impuissance : anticiper, transmettre dans la transparence, poser ses limites, faire la place à l'autre, orienter en gardant sa place...

Dans le passé, toxicomanie et incapacité parentale se mariaient aisément dans l'esprit des professionnels, en parti- culier ceux qui doivent veiller à la sécurité des liens familiaux. Il a fallu apprendre à différencier les registres dans ce qu'on appelle « lien mère-enfant ». Les sentiments maternels sont une chose, les capacités émotionnelles et cognitives permettant de s'ajuster aux besoins d'un nourrisson (caregiving) en sont une autre, la sensation éprouvée d'être reconnue dans son identité parentale une troisième. Tout le travail vise alors à ne pas entraver les premier et troisième niveaux, à partir de quoi la mère (et/ou le père) peut identifier ses propres difficultés éducatives en y étant accompagné. Il s'agit d'un travail d'équipe, aucun professionnel ne peut en saisir seul la complexité. L'objectif n'est pas de maintenir à tout prix l'enfant au domicile dans les cas de grande fragilité, mais au contraire d'organiser avec les parents les conditions de la sécurité tant de l'enfant que d'eux-mêmes. Plus on se rapproche des parents à l'occasion des soins périnataux, mieux ils accepteront une distance parfois nécessaire avec leur enfant.

Sur tous ces aspects, l'expérience acquise permet d'appliquer les mêmes principes dans les situations de troubles psychiques maternels qui, associés à la grossesse, font désormais l'objet de protocoles ou guides de bonnes pratiques : un cadre interdisciplinaire centré sur le suivi obstétrical permet l'engagement coordonné des équipes obstétricales, pédiatriques, psychiatriques, médicosociales. Peu à peu s'organise la complexité d'un suivi global jusqu'alors morcelé dans le temps au travers d'interventions issues de champs différents, sans communication entre eux. Les principes et les outils existent, il reste à les mettre en œuvre : pour ne citer qu'un exemple la liaison personnalisée entre médecin traitant, obstétricien, sage-femme et psychiatre balbutie encore.

Dans l'histoire des sciences, toute avancée permet d'élaborer une théorie descriptive, repère de travail provisoire, dont on sait qu'elle ouvre un nouveau champ d'inconnu. Là où nous en sommes en effet, des questions inédites surgissent : comment articuler les places, définir les complémentarités et les niveaux d'intervention, lorsqu'il s'agit d'intégrer dans un même projet l'ensemble des données somatiques, sociales, psycho-émotionnelles ? Les débats actuels sont traversés de questions aiguës touchant aux identités et cultures professionnelles. Le simple fait, acquis dans certaines équipes, que la grande vulnérabilité psychologique, génératrice de stress, justifie un suivi de type « grossesse à haut risque », n'est pas admis partout, malgré les publications sur les effets périnataux du désordre émotionnel. La fonction de coordination, évidente à tel endroit dans les cas complexes, ne s'imposera pas ailleurs. S'agit-il de collaborer entre médecin et sage-femme, ou d'identifier sur tel niveau de risque une place de sage-femme coordinatrice pour tenir la cohérence de l'ensemble ? Le médecin a-t-il les moyens de coordonner lui-même la diversité des interventions ? Le psychologue doit-il assurer ce rôle, y est-il 
reconnu par l'usager? La globalité vue par le professionnel est-elle la même que celle vécue par les parents ? La prise en compte du réseau de proximité doit-elle réduire le rôle pourtant essentiel du passage en maternité - comme on l'entend parfois lors des revues de dossiers complexes? Qui appelle qui, et à quel moment ? Comment les praticiens de la grossesse/naissance tiennent-ils dans les situations relationnelles éprouvantes ? Quelles leçons en tirer pour l'efficacité de l'intervenant «psy »?

Nouvelles pratiques, nouvelles questions. Depuis juin 1998, un groupe pluriprofessionnel de réflexion et de recherche s'est mis en place autour de la prise en charge des femmes enceintes abusant de substances psycho-actives et de leurs enfants ; à ce jour, une cinquantaine d'équipes pluridisciplinaires françaises y collaborent (obstétriciens, sages-femmes, pédiatres, psychologues, psychiatres, pédopsychiatres, déontologistes, addictologues, pharmacologues...). L'acte constitutif de ce groupe a été la participation à une étude multicentrique entre 1998 et 1999 coordonnée par le Pr Claude Lejeune qui a abouti à la publication des résultats de la plus grande série française de femmes enceintes ex-toxicomanes aux opiacés sous traitement de substitution et de leurs nouveau-nés [4]. Constitué en association loi 1901, le Groupe d'étude grossesse et addictions (GEGA) porte depuis lors des projets de recherche et organise des journées thématiques, offrant ainsi un espace de formation et d'échanges pluridisciplinaires. Il exerce un rôle d'expert auprès de l'ANAES en 2004, puis l'HAS en 2007 et la MITDT en 2011 [5].

Depuis 2008, le GEGA a promu la création d'un Diplôme d'université "Périnatalité et addictions » à la faculté de médecine de l'université Paris-Diderot. Cet enseignement a évolué vers un diplôme interuniversitaire depuis 2012 avec les universités de Nantes, Montpellier et Strasbourg. L'objectif de la formation est d'améliorer les compétences de tous les professionnels impliqués dans la prise en charge des femmes enceintes abusant de substances psycho-actives et de leurs enfants et de valoriser le travail multidisciplinaire et en réseau, pour favoriser la prévention des complications périnatales et améliorer le pronostic de ces femmes, de leurs enfants et des familles. Il est destiné à tout professionnel des champs médical, social et judiciaire concernés par la consommation de substances psycho-actives pendant la grossesse et ses conséquences. La réflexion se poursuit ainsi d'année en année sur les interrogations qui surgissent au fur et à mesure d'une avancée dans les pratiques.

En 2013, le RESPADD (Réseau des établissements de santé pour la prévention des addictions) a traduit et publié un ouvrage collectif [6] qui rend compte des pratiques de terrain actualisées. La première version anglaise coordonnée par A. Whittaker a été enrichie de nombreuses contributions issues du GEGA et de la Fédération « Addiction » afin qu'il réponde aux besoins, préoccupations et spécificités françaises.

\section{Références}

1. Chanal C, Mazurier E, Benos P, et al (2000) Enceinte et toxicomane. DVD Afree www.afree.asso.fr (accompagne l'ouvrage " Grossesse et toxicomanie ", coord F. Molénat, éd Erès 2000)

2. Roy J, Toubin RM, Mazurier E, et al (2001) Devenir à 5 ans des enfants de mères dépendantes aux opiacés : effets d'un suivi multidisciplinaire pendant la grossesse. Arch Ped 18:1130-8

3. Réseau NGLR (2012) Fiches techniques « consommations de substances psychoactives pendant la grossesse ». http://www.perinatfrance.org/portail-professionnel/referentiels/protocoles-regionaux/ languedoc-roussillon/perinatalite-et-addictions-645-1929.html

4. Lejeune C, Simmat-Durand L, Gourarier L, et al (2006) Prospective multicenter observationnal study of 260 infants born to 259 opiate dependant mothers on methadone or high-dose buprenorphine substitution. Drug Alcohol Depend 82:250-7. http://www.asso-gega. org/spip.php?rubrique1

5. Whittaker A (2013) Drugscope. Guide concernant l'usage de substances psychoactives durant la grossesse. Trad. française coord RESPADD, $336 \mathrm{p}$

6. Baumler M, Kojayan K, Toubin RM, et al (2007) Grossesse et troubles psychiatriques, recommandations de bonnes pratiques. $37^{\text {es }}$ Journées de la Société de médecine périnatale. Arnette 45-62 